\title{
ZC3H7B-BCOR high-grade endometrial stromal sarcomas: a report of 17 cases of a newly defined entity
}

Natasha Lewis ${ }^{1}$, Robert A Soslow ${ }^{1}$, Deborah F Delair ${ }^{1}$, Kay J Park ${ }^{1}$, Rajmohan Murali ${ }^{1}$, Travis J Hollmann ${ }^{1}$, Ben Davidson ${ }^{2,3}$, Francesca Micci ${ }^{4}$, Ioannis Panagopoulos ${ }^{4}$, Lien N Hoang ${ }^{5}$, Javier A Arias-Stella III ${ }^{1}$, Esther Oliva ${ }^{6,7}$, Robert H Young ${ }^{6,7}$, Martee L Hensley ${ }^{8}$, Mario M Leitao Jr ${ }^{9}$, Meera Hameed ${ }^{1}$, Ryma Benayed ${ }^{\mathrm{P}}$, Marc Ladanyi ${ }^{1}$, Denise Frosina $^{1}$, Achim A Jungbluth ${ }^{1}$, Cristina R Antonescu ${ }^{1}$ and Sarah Chiang ${ }^{1}$

${ }^{1}$ Department of Pathology, Memorial Sloan Kettering Cancer Center, New York, NY, USA; ${ }^{2}$ Department of Pathology, The Norwegian Radium Hospital, Oslo University Hospital, Oslo, Norway; ${ }^{3}$ Faculty of Medicine, University of Oslo, Oslo, Norway; ${ }^{4}$ Section for Cancer Cytogenetics, Institute of Cancer Genetics and Informatics, The Norwegian Radium Hospital, Oslo University Hospital, Oslo, Norway; ${ }^{5}$ Department of Pathology, Vancouver General Hospital, Vancouver, BC, Canada; ${ }^{6}$ Department of Pathology, Massachusetts General Hospital, Boston, MA, USA; ${ }^{7}$ Department of Pathology, Harvard Medical School, Boston, MA, USA; ${ }^{8}$ Gynecologic Medical Oncology Service, Department of Medicine, Memorial Sloan Kettering Cancer Center, New York, NY, USA and ${ }^{9}$ Gynecology Service, Department of Surgery, Memorial Sloan Kettering Cancer Center, New York, NY, USA

High-grade endometrial stromal sarcoma likely encompasses underrecognized tumors harboring genetic abnormalities besides YWHAE-NUTM2 fusion. Triggered by three initial endometrial stromal sarcomas with ZC3H7B-BCOR fusion characterized by high-grade morphology and aggressive clinical behavior, we herein investigate the clinicopathologic features of this genetic subset by expanding the analysis to 17 such tumors. All of them occurred in adult women with a median age of 54 (range, 28-71) years. They were predominantly based in the endomyometrium and demonstrated tongue-like and/or pushing myometrial invasion. Most were uniformly cellular and displayed haphazard fascicles of spindle cells with mild to moderate nuclear atypia. Myxoid matrix was seen in 14 of $17(82 \%)$ tumors, and collagen plaques were seen in $8(47 \%)$. The mitotic index was $\geq 10$ mitotic figures/10 high-power fields (HPFs) in 14 of 17 (82\%) tumors with a median of 14.5 mitotic figures/10 HPFs. No foci of conventional or variant low-grade endometrial stromal sarcoma were seen. All tumors expressed CD10 with only limited or absent desmin, SMA and/or h-caldesmon staining. ER and PR expression in $>5 \%$ of cells was seen in 4 of $12(33 \%)$ tumors. Diffuse cyclin D1 and BCOR immunoreactivity was present in 7 of $8(88 \%)$ and 7 of $14(50 \%)$ tumors, respectively. Fluorescence in situ hybridization or targeted RNA sequencing confirmed ZC3H7B-BCOR fusion in all tumors, including four and two previously diagnosed as myxoid leiomyosarcoma and undifferentiated uterine sarcoma, respectively. Limited clinical data suggest that patients present at higher stage and have worse prognosis compared with published outcomes in low-grade endometrial stromal sarcoma. Tumors with $Z C 3 H 7 B-B C O R$ fusion constitute a distinct group of endometrial stromal sarcomas with high-grade morphology that should be distinguished from other uterine mesenchymal neoplasms that may demonstrate myxoid morphology.

Modern Pathology (2018) 31, 674-684; doi:10.1038/modpathol.2017.162; published online 1 December 2017

In the most recent World Health Organization classification of endometrial stromal tumors, the

Correspondence: Dr S Chiang, MD, Department of Pathology, Memorial Sloan Kettering Cancer Center, 1275 York Avenue, New York 10065, NY, USA.

E-mail: chiangs@mskcc.org

Received 5 September 2017; revised 19 September 2017; accepted 14 October 2017; published online 1 December 2017 term 'high-grade endometrial stromal sarcoma' describes neoplasms harboring the chromosomal translocation $\mathrm{t}(10 ; 17)(\mathrm{q} 22 ; \mathrm{p} 13)$ resulting in YWHAE-NUTM2 gene fusion. ${ }^{1-3}$ High-grade endometrial stromal sarcomas with YWHAE-NUTM2 fusion have unique histologic features, typically exhibiting a high-grade round cell component that is sometimes associated with a low-grade fibrous or fibromyxoid spindle cell component ${ }^{4,5}$ or may rarely 
evolve from a conventional low-grade endometrial stromal sarcoma. ${ }^{6}$ The round cell component consists of monomorphic cells with intermediate-sized nuclei and finely dispersed chromatin, arranged in sheets and nests with pseudo-papillary or pseudoglandular architecture and associated with brisk mitotic activity., ${ }^{4,5}$ This component lacks CD10, ER and PR expression and exhibits strong and diffuse cyclin D1 and BCOR staining. ${ }^{8,9}$ Compared with patients with low-grade endometrial stromal sarcoma, those with YWHAE-NUTM2 high-grade endometrial stromal sarcoma have earlier and more frequent recurrences and are more likely to die of their disease..$^{2,4,7,10}$ Overall, clinical outcomes appear intermediate between low-grade endometrial stromal sarcoma and undifferentiated uterine sarcoma. ${ }^{4,11}$

Although YWHAE-NUTM2 high-grade endometrial stromal sarcoma is now readily recognizable by its characteristic morphologic features, other types of high-grade endometrial stromal sarcomas with alternative gene fusions likely exist and may currently be misclassified as undifferentiated uterine sarcoma or leiomyosarcoma. ${ }^{7,12}$ ZC3H7B-BCOR gene fusion resulting from the chromosomal translocation $\mathrm{t}(\mathrm{X} ; 22)(\mathrm{p} 11 ; \mathrm{q} 13)$ was recently identified in both ossifying fibromyxoid tumors ${ }^{13}$ and endometrial stromal sarcomas, ${ }^{14}$ the latter in which tumor grade was not specified. Endometrial stromal sarcomas harboring this novel fusion appear to share histologic and some immunophenotypic features with myxoid leiomyosarcoma. ${ }^{12}$ Our initial study of three such tumors suggested that they are histologically high-grade because of the presence of both brisk mitotic activity and nuclear atypia exceeding that allowable for low-grade endometrial stromal sarcoma. ${ }^{12}$ Furthermore, the limited clinical data available suggested that $Z C 3 H 7 B-B C O R$ endometrial stromal sarcomas may be clinically aggressive compared with low-grade endometrial stromal sarcoma. ${ }^{12}$

In this study, we describe the histologic and immunophenotypic features of the largest cohort of $Z C 3 H 7 B-B C O R$ endometrial stromal sarcomas to date to define these tumors as a distinct pathologic entity that can and should be distinguished from other uterine mesenchymal tumors, particularly myxoid leiomyosarcoma, and evaluate their clinical behavior.

\section{Materials and methods}

\section{Case Selection}

Seventeen endometrial stromal sarcomas harboring $Z C 3 H 7 B-B C O R$ fusions were collected from four institutions: Memorial Sloan Kettering Cancer Center, New York, NY, USA ( $n=10)$; Norwegian Radium Hospital, Oslo, Norway $(n=3)$; Vancouver General Hospital, Vancouver, Canada $(n=2)$; Massachusetts
General Hospital, Boston, MA, USA $(n=1)$; and the pathology consultation files of one of the authors (RHY) $(n=1)$. $Z C 3 H 7 B-B C O R$ fusion was identified in 12 tumors, 5 of which were previously reported, ${ }^{12,14}$ as part of the clinical work-up using fluorescence in situ hybridization (FISH) or nextgeneration sequencing platforms including Archer FusionPlex Custom Solid Panel, MSK-Integrated Mutation Profiling of Actionable Cancer Targets (MSK-IMPACT), which interrogates all exons and selected regulatory regions and introns of $410 \mathrm{key}$ cancer genes; ${ }^{15}$ and FoundationOne, a genomic profiling assay interrogating the entire coding sequence of 315 cancer-related genes and selected introns from 28 genes often rearranged or altered in solid tumors. The remaining five tumors were identified by searching the Memorial Sloan Kettering Cancer Center clinical and pathology databases and the Vancouver General Hospital pathology database using the following search terms: 'undifferentiated uterine sarcoma,' 'undifferentiated endometrial sarcoma' and 'myxoid leiomyosarcoma.' Five- $\mu \mathrm{m}$, formalin-fixed, paraffin-embedded tumoral tissue sections of these five cases were subjected to FISH and/or Archer FusionPlex sequencing. Four and two tumors were previously diagnosed as myxoid leiomyosarcoma and undifferentiated uterine sarcoma, respectively.

All available hematoxylin-and-eosin (H\&E) and immunohistochemical stained slides and pathology reports of $Z C 3 H 7 B-B C O R$ endometrial stromal sarcomas were reviewed by two pathologists (SC and NL) at a multi-head microscope for confirmation of diagnosis. Gross, morphologic and immunophenotypic features of these tumors were recorded. Clinical information, including age, stage and outcome, was obtained through the electronic medical records and pathology reports at Memorial Sloan Kettering Cancer Center, Massachusetts General Hospital, Vancouver General Hospital and Norwegian Regional Hospital. The study was approved by institutional review boards.

\section{Fluorescence In Situ Hybridization}

FISH using probes flanking YWHAE, BCOR and $Z C 3 H 7 B$ genes was performed on $5-\mu \mathrm{m}$ formalinfixed, paraffin-embedded whole tissue sections of tumors with unknown gene rearrangement status as previously described. ${ }^{12,16}$ Custom probes were made by bacterial artificial chromosomes (BAC) clones flanking the YWHAE (RP11-105D11, RP11-1142D6, RP11-170J13, RP11-806J5), BCOR (RP11-21D3, RP111105N2, RP11-37K20, RP11-973F20) and $Z C 3 H 7 B$ (RP11-1078O11, RP11-110H11) genes and obtained from BAC/PAC Resources (Children's Hospital Oakland Research Institute, Oakland, CA, USA). ${ }^{13,16}$ BAC clones were labeled with nick translation and validated on normal metaphase chromosomes. Slides were deparaffinized, pretreated and 
hybridized with denatured probes overnight, followed by post-hybridization washes and counterstaining with DAPI. Slides were examined on a Zeiss fluorescence microscope (Zeiss Axioplan, Oberkochen, Germany) using Isis 5 software (Metasystems). Two hundred tumor nuclei were counted, and gene rearrangement was confirmed if break-apart signals were seen in $>20 \%$ of nuclei.

\section{Next-Generation Sequencing}

Samples were subjected to the Archer FusionPlex Custom Solid Panel (ArcherDX Inc., Boulder, CO, USA), a targeted RNA sequencing assay utilizing the Anchored Multiplex PCR (AMP) technology that detects gene fusions and oncogenic isoforms in selected protein-coding exons of 62 genes. ${ }^{17}$ Tumor RNA was extracted from $5-\mu \mathrm{m}$ formalin-fixed, paraffin-embedded tissue sections followed by cDNA synthesis and library preparation. Final targeted amplicons were sequenced on an Illumina MiSeq. Data were analyzed using the Archer Software (version 4.0.10; ArcherDX Inc.).

\section{Immunohistochemistry}

Immunohistochemical staining was performed on $5-\mu \mathrm{m}$ whole tissue sections of $Z C 3 H 7 B-B C O R$ endometrial stromal sarcomas with available material using the following antibodies: CD10, ER, PR, smooth muscle actin (SMA), desmin, h-caldesmon, cyclin D1 and BCOR (Table 1). For the remaining cases without additional available material, immunohistochemical stain results were recorded based on review of slides and/or extracted from pathology reports submitted by referring institutions.

\section{Results}

\section{Gross and Microscopic Features}

Tumor size was recorded in 16 of 17 ZC3H7B-BCOR endometrial stromal sarcomas and ranged from 1.5 to 12.0 (median, 9.7) $\mathrm{cm}$. Size was not available in the remaining case in which the diagnosis was made on an endometrial curettage, and a hysterectomy was not performed. Additional gross characteristics were available in eight tumors: five were polypoid, tan to pink to yellow, solid, soft, fleshy or rubbery masses that involved the endometrium (Figure 1); one tumor appeared grossly confined to the myometrium, but had irregular borders; one was received fragmented and one was morcellated, precluding reliable assessment of endomyometrial involvement.

Histologically, the tumors more often involved both the endometrium and myometrium (11 cases) than myometrium only (2 cases). Two patterns of myometrial invasion were observed among the 12 neoplasms in which the tumor border was available for evaluation: a tongue-like, infiltrative pattern typical of low-grade endometrial stromal sarcoma seen in five tumors (Figure 2a) and a broad front with irregular borders in two (Figure 2b); five displayed

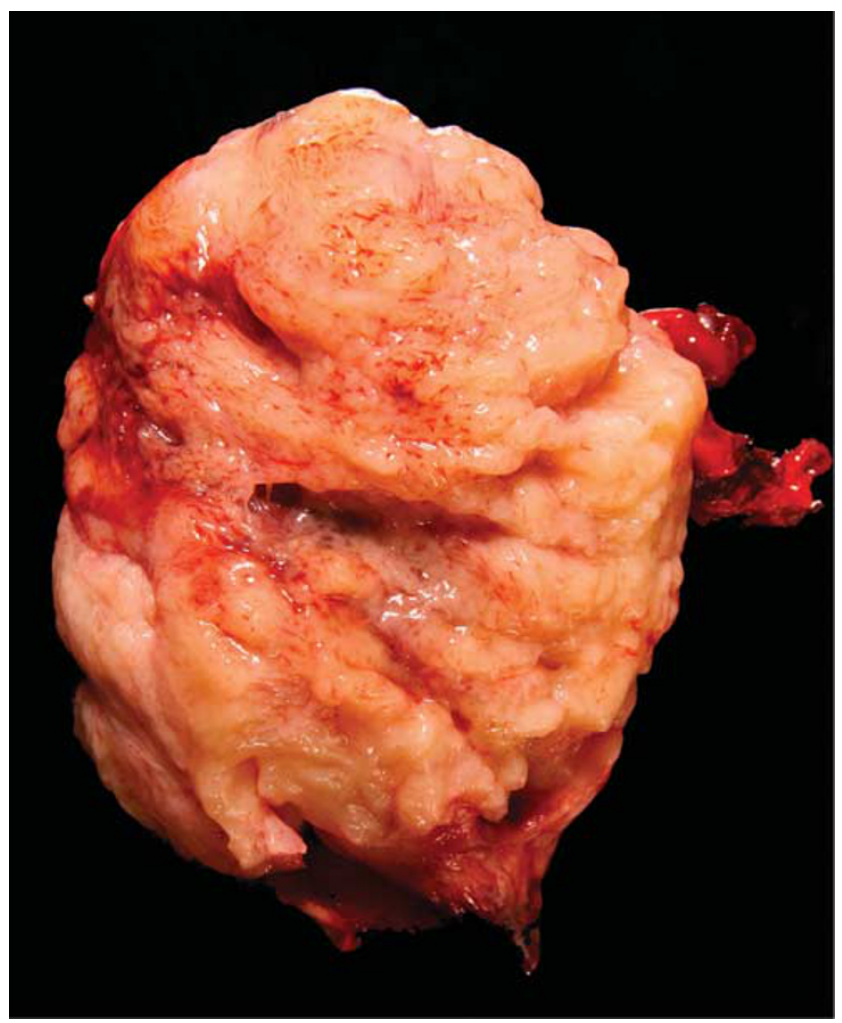

Figure 1 Gross features of $Z C 3 H 7 B-B C O R$ endometrial stromal sarcoma. A yellow and tan-white, fungating mass occupies the entire endometrial cavity.

Table 1 Antibodies used for immunohistochemistry

\begin{tabular}{lcllcccc}
\hline \multicolumn{1}{c}{ Antibody } & Clone & Vendor & Detection kit & Dilution & Retrieval (min) & Incubation (min) & Instrument \\
\hline CD10 & SP67 & Roche & Optiview & RTU & 64 CC1 & 24 & Roche Benchmark Ultra \\
ER & 6F11 & Leica & Bond Polymer & RTU & 25 ER1 & 20 & Leica Bond III \\
PR & 16 & Leica & Bond Polymer & RTU & 20 ER2 & 15 & Leica Bond III \\
SMA & 1A4 & Cell Marque & Optiview & $1: 200$ & None & 32 & Roche Benchmark \\
Desmin & DER11 & Roche & Optiview & RTU & 24 CC1 & 20 & Roche Benchmark Ultra \\
H-caldesmon & E89 & Cell Marque & Optiview & RTU & 24 CC1 & 20 & Roche Benchmark \\
Cyclin D1 & RM-9104- S & Thermo & Optiview & $1: 100$ & 32 CC1 & 32 & Roche Benchmark \\
BCOR & C-10 & Santa Cruz & Bond Polymer & $1: 150$ & 30 ER2 & 30 & Leica Bond III \\
\hline
\end{tabular}

Abbreviations: CC1, cell conditioning 1; ER, epitope retrieval; RTU, ready to use. 

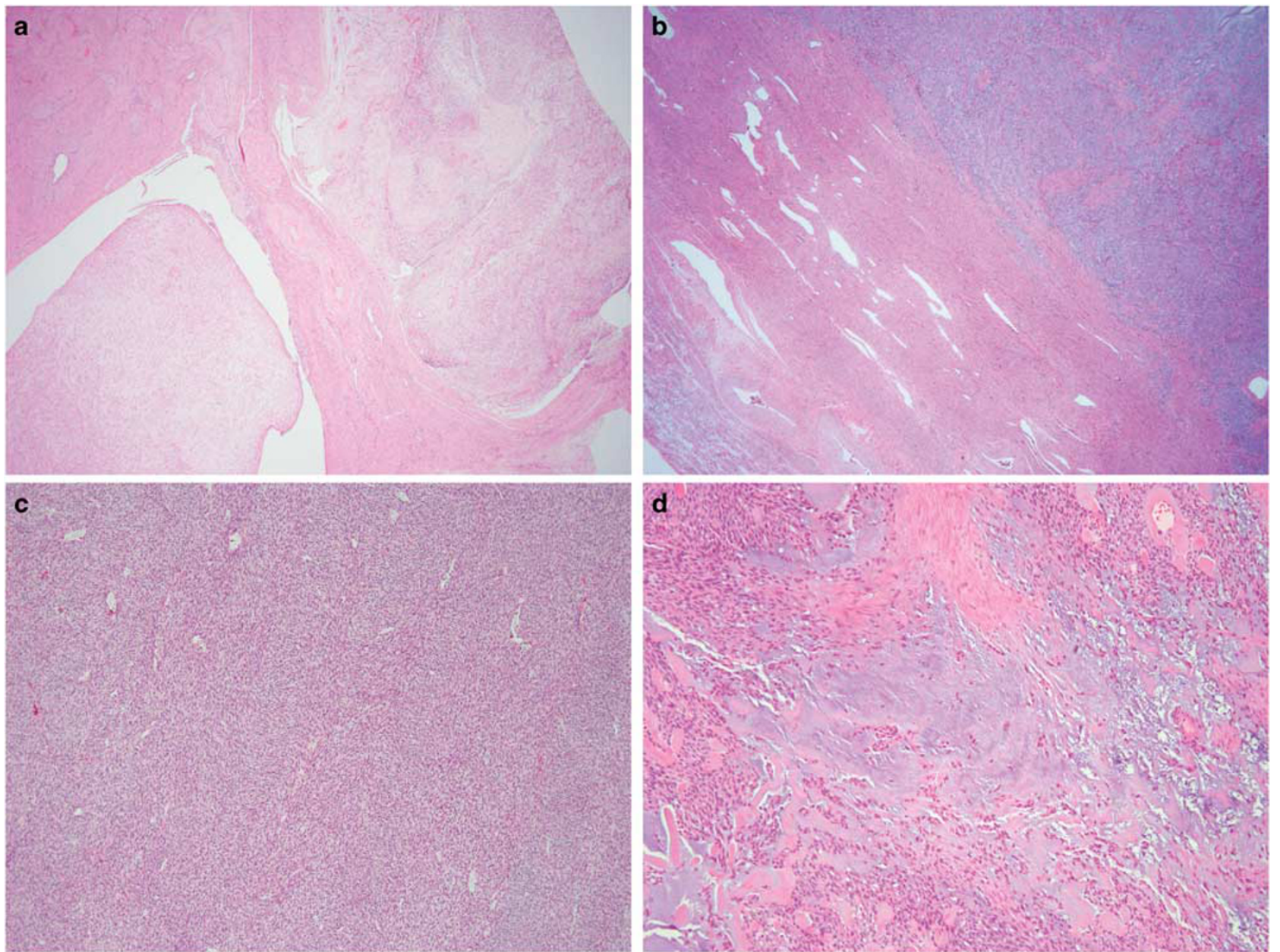

Figure 2 Histologic features of ZC3H7B-BCOR endometrial stromal sarcoma. Tongue-like, infiltrative growth patterns with lymphovascular invasion typical of endometrial stromal sarcomas (a) is more common than myometrial invasion with a broad but irregular border (b). Haphazard fascicular architecture (c) and myxoid stroma (d) were also common.

both patterns. Necrosis was seen in 10 of $17(59 \%)$ tumors and was of infarct-type in 8 tumors and tumor-type in 1; 1 tumor demonstrated both infarct and tumor cell necrosis. Lymphovascular invasion was common, present in 11 of 17 (64\%) cases.

The tumors were uniformly cellular and grew in haphazard fascicles (Figure 2c) composed of spindle cells, which had intermediate-sized ovoid to spindle and occasionally round nuclei with an even chromatin pattern and inconspicuous or 1-3 pinpoint nucleoli, except in one case that showed marked nuclear pleomorphism. Cytoplasm ranged from scant to moderate and eosinophilic (12 cases) to more abundant and blue-gray (4 cases). Myxoid stroma was identified in 14 of $17(82 \%)$ tumors (Figure 2d); it was abundant in 4, forming lakes, and focal in 6. Collagen plaques were seen in eight tumors and were extensive in one (Figure 3a). Scattered round, 'ball-like' proliferations of spindle cells were seen in one tumor (Figure $3 b$ ), and rare benign endometrioid glands in two others (Figure 3c). The mitotic rate ranged from 1 to 50 mitotic figures/10 high-power fields (HPF) with a median of 14.5 mitotic figures/10 HPF. Fourteen $(82 \%)$ tumors had mitotic indices of $\geq 10$ mitotic figures/10 HPF. The vascular pattern was variable, with a single case demonstrating virtually no significant vascularity, 10 tumors showing small arterioles without prominent perivascular tumor cell whorling (Figure 3d), 1 exhibiting occasional larger, thick-walled vessels (Figure 3e) and 2 containing both small arterioles and thick-walled vessels. Three tumors displayed occasional hemangiopericytoma-like dilated vessels in addition to either small arterioles or thick-walled vessels (Figure 3f). Rare benign endometrioid glands were present in two tumors (Figure 3c); however, no foci of conventional or variant low-grade endometrial stromal sarcoma were seen in any of the neoplasms in the entire cohort.

\section{Immunohistochemical Findings}

The immunohistochemical profile of the neoplasms is summarized in Table 2 and illustrated in Figure 4. CD10 was positive in all $17(100 \%)$ tumors. The 

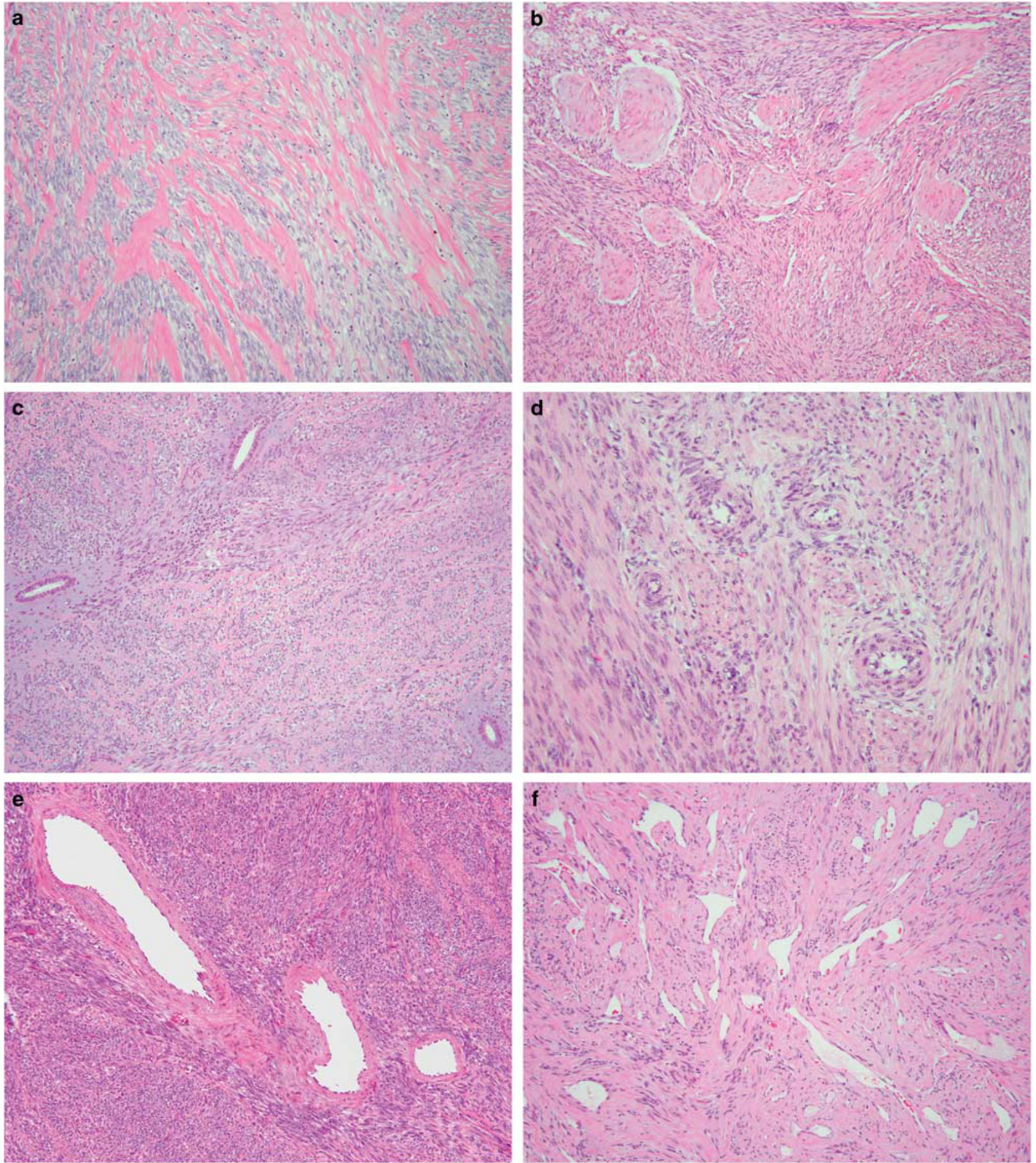

Figure 3 Additional morphologic features seen among some ZC3H7B-BCOR endometrial stromal sarcomas included collagen plaques (a), round, 'ball-like' proliferations of spindled cells (b), scattered benign endometrioid glands (c), small arterioles without prominent perivascular tumor cell whorling (d), larger, thick-walled blood vessels (e) and hemangiopericytoma-like dilated blood vessels without perivascular whorling (f).

staining intensity and extent were typically strong and diffuse with three (19\%) tumors demonstrating focal and/or weak expression. Only 7 of 16 (44\%) tumors showed limited evidence of myogenic differentiation, including 6 demonstrating focal staining with only one smooth muscle marker (5 SMA and 1 desmin) and 1 expressing both SMA and h-caldesmon, but lacking desmin immunoreactivity. Smooth muscle markers were negative in the remaining nine $(56 \%)$ tumors. ER and PR were also variably 
Table 2 Immunophenotype of $Z C 3 H 7 B-B C O R$ endometrial stromal sarcomas

\begin{tabular}{|c|c|c|c|c|c|c|c|c|}
\hline Case & CD10 & $E R$ & $P R$ & $S M A$ & Desmin & H-caldesmon & Cyclin D1 & BCOR \\
\hline 1 & Pos & Pos & Pos & Neg & Neg & ND & Pos $(S, D)$ & Pos $(S,>95 \%)$ \\
\hline 2 & Pos & ND & ND & Neg & Pos (F) & Neg & Neg & Neg \\
\hline 3 & Pos & Pos & Pos & Neg & ND & ND & ND & Neg \\
\hline 4 & Pos (F) & Neg & ND & Neg & Neg & Neg & Pos (M) & Pos $(W,>95 \%)$ \\
\hline 5 & Pos & ND & ND & Pos & Neg & Pos & Pos (S,D) & Pos (W, $<5 \%)$ \\
\hline 6 & Pos & ND & ND & Pos (F) & Neg & Neg & ND & $\operatorname{Pos}(\mathrm{S},>95 \%)$ \\
\hline 7 & Pos & ND & ND & ND & ND & ND & ND & Neg \\
\hline 8 & Pos & Neg & Neg & ND & Neg & ND & ND & $\operatorname{Pos}(\mathrm{S},>95 \%)$ \\
\hline 9 & Pos (W) & Neg & Neg & Neg & Neg & Neg & ND & Pos (W, $<5 \%)$ \\
\hline 10 & Pos & Neg & Neg & Neg & Neg & Neg & Pos (S,D) & Pos $(S,>95 \%)$ \\
\hline 11 & Pos & Pos & Pos & Pos (F) & Neg & ND & Pos (D) & $\operatorname{Pos}(\mathrm{M},<5 \%)$ \\
\hline 12 & Pos & Neg & Neg & Neg & Neg & Neg & ND & ND \\
\hline 13 & Pos & Neg & Neg & Pos (F) & Neg & Neg & ND & ND \\
\hline 14 & Pos & Pos & Pos & Neg & Neg & Neg & ND & ND \\
\hline 15 & Pos & Neg & Neg & Pos (F) & Neg & Neg & Pos & Pos $(S,>95 \%)$ \\
\hline 16 & Pos (F) & Neg & ND & Pos (F) & Neg & ND & Pos (S,D) & Pos (S, 25\%) \\
\hline 17 & Pos & ND & ND & Neg & Neg & Neg & ND & $\operatorname{Pos}(\mathrm{S},>95 \%)$ \\
\hline
\end{tabular}

Abbreviations: D, diffuse; ER, estrogen receptor; F, focal; M, moderate; ND, not done; Neg, negative; Pos, positive; PR, progesterone receptor; S, strong; SMA, smooth muscle actin; W, weak.

expressed, demonstrating positive staining for both markers in $>5 \%$ of cell in only 4 of $13(31 \%)$ cases. Cyclin D1 was strong and diffuse ( $>95 \%$ of tumor cells) in seven of eight (88\%) tumors. BCOR immunohistochemical staining was performed on 14 tumors and showed diffuse ( $>95 \%$ of tumor cells) expression that was strong in $6(43 \%)$ and weak in $1(7 \%)$ tumor. BCOR expression was strong in $\sim 25 \%$ of the cells in one $(7 \%)$, weak to moderate in $<5 \%$ of the cells in three $(21 \%)$ and completely negative in three $(21 \%)$ tumors. Among the eight tumors for which both BCOR and cyclin D1 were performed, six showed concordant staining patterns, meaning, individual cases either dually expressed both markers or were negative for both. Only two tumors showed diffuse cyclin D1, but only focal BCOR expression.

\section{Clinical Data Including Follow-Up}

Age and stage information using the 2009 FIGO staging system for uterine sarcomas was collected for all 17 cases. The median age at diagnosis was 54 (range $28-71)$ years. Seven $(41 \%)$, three $(18 \%)$ and seven $(41 \%)$ patients presented with FIGO stage 1, 2 and 3 disease, respectively. Of the five patients who underwent lymph node sampling, two had lymph node metastases, one of whom had extrauterine disease at presentation, whereas the other had tumor otherwise limited to the uterus.

Clinical records of the $10 Z C 3 H 7 B-B C O R$ endometrial stromal sarcoma patients from Memorial Sloan Kettering Cancer Center were reviewed. Five patients were seen only once in consultation, three of whom at the time of recurrence. Among the five patients with clinical follow-up, only two were seen soon after initial diagnosis. One presented with stage 3 disease and had a pelvic recurrence that was surgically resected 3 months after initial diagnosis; the patient had no evidence of disease 12 months later. The other patient also had stage 3 disease at presentation and subsequently developed an abdominal/pelvic recurrence after 19 months that progressed despite medical management; she died 21 months later with an overall survival of 40 months. The three remaining patients were all seen at the time of recurrence, treated only with nonsurgical options, and had persistent and progressive disease. These patients presented with stage 1 disease, and all died of disease with overall survivals of 34, 41 and 80 months, respectively, after initial diagnosis.

\section{Discussion}

This is the largest study to date of $Z C 3 H 7 B-B C O R$ fusion-positive endometrial stromal sarcomas, in which we have presented a description of the histologic, immunohistochemical and clinical features of this rare uterine sarcoma. These tumors often involve the endometrium and invade the myometrium in a tongue-like and/or pushing pattern. They consist of haphazard fascicles of spindle cells embedded in variable amounts of myxoid matrix and are frequently associated with brisk mitotic activity. They typically express CD10 and exhibit either limited or absent smooth muscle differentiation by immunohistochemistry; diffuse cyclin D1 and BCOR staining is seen in at least half of them. In contrast to patients with low-grade endometrial stromal sarcoma who typically have an indolent clinical course, ${ }^{1}$ those with $Z C 3 H 7 B$-BCOR endometrial stromal sarcoma tend to present with higher stage disease, develop recurrence sooner and die of disease more frequently, similar to reported outcomes of patients with YWHAE-NUTM2 high-grade endometrial stromal sarcoma. ${ }^{2,4}$ ZC3H7B-BCOR endometrial stromal sarcoma represents a 

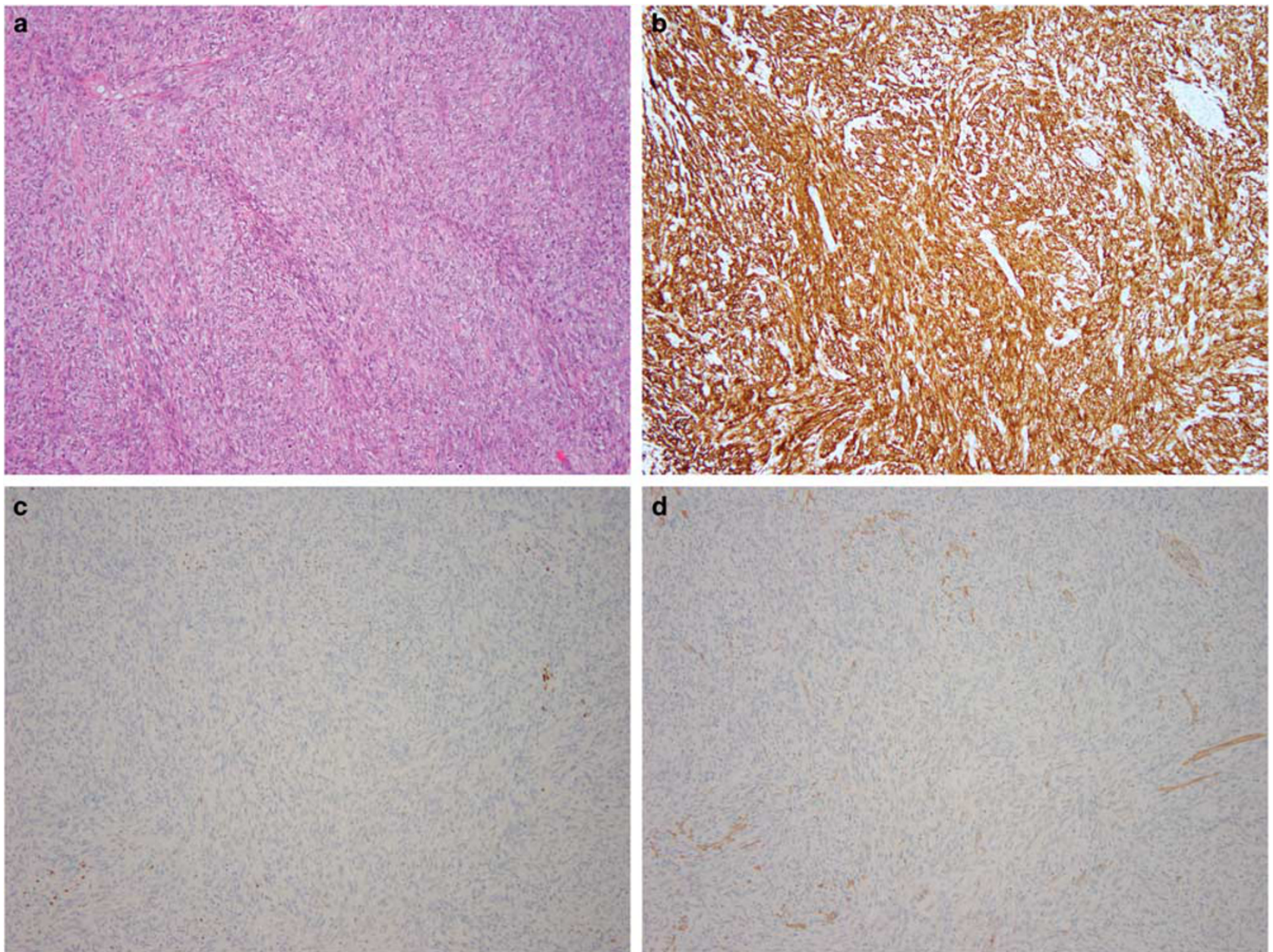

d
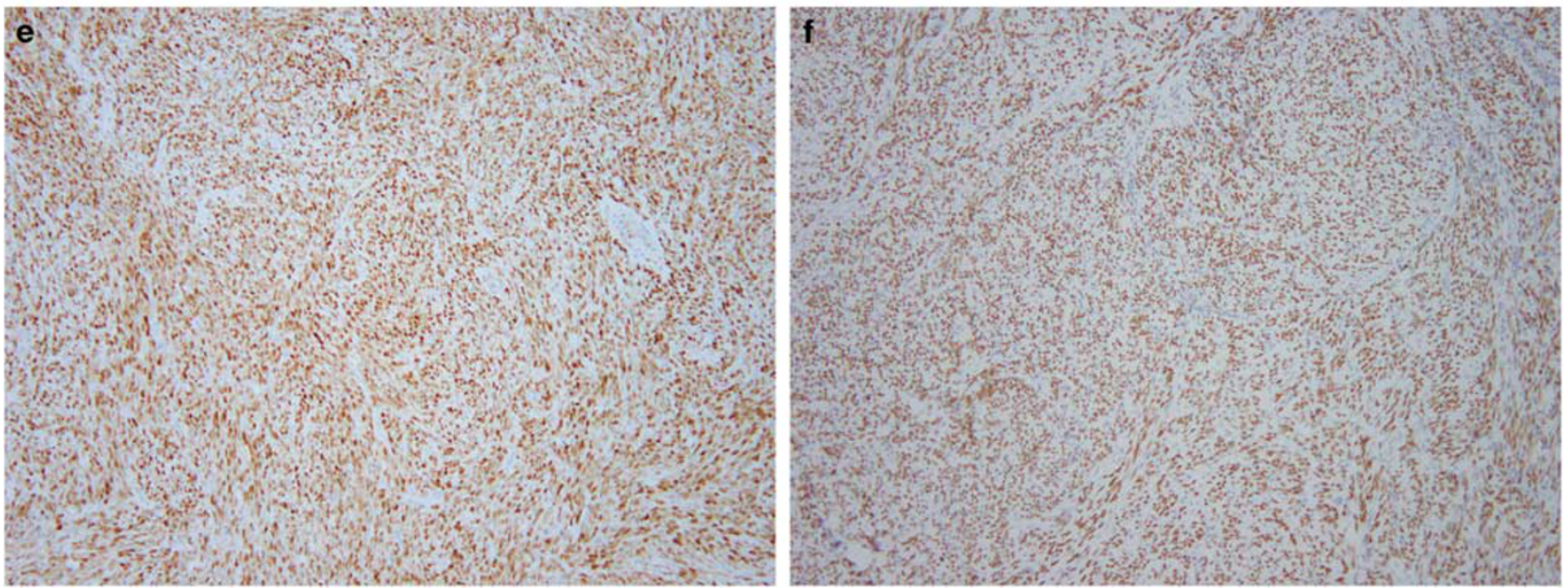

Figure 4 H\&E (a) and CD10 (b), ER (c), SMA (d), cyclin D1 (e) and BCOR (f) immunohistochemical stains from a ZC3H7B-BCOR endometrial stromal sarcoma. The immunoprofile was typical of the cohort, showing strong and diffuse reactivity for CD10, cyclin D1 and BCOR while demonstrating focal, weak positivity for ER and SMA.

histologically high-grade neoplasm that requires distinction from other uterine mesenchymal tumors that may exhibit myxoid morphology.

Based on the findings from our current and recently published studies, ${ }^{12} Z C 3 H 7 B-B C O R$ endometrial stromal sarcoma represents a unique clinicopathologic entity in which a recurrent gene fusion is associated with distinctive histology. The majority of these tumors arise in the endometrium and exhibit tongue-like myometrial infiltration and lymphovascular invasion similar to low-grade and YWHAENUTM2 high-grade endometrial stromal sarcomas. However, in contrast to low-grade endometrial stromal sarcoma, tumor cells of ZC3H7B-BCOR 
endometrial stromal sarcoma demonstrate a greater degree of nuclear atypia, are arranged in haphazard fascicles without perivascular whorling, and frequently show myxoid matrix. Tumors may also demonstrate a broad front of myometrial invasion with irregular borders different from the typical invasion pattern of endometrial stromal sarcomas. Collagen plaques, which are common among lowgrade endometrial stromal sarcomas, are present in approximately half of tumors harboring $Z C 3 H 7 B$ $B C O R$ fusion. However, foci of conventional or variant low-grade endometrial stromal sarcoma morphology are not seen in these tumors. The distinctive morphology, degree of cytologic atypia associated with brisk mitotic activity and lack of any morphologic resemblance to low-grade endometrial stromal sarcomas together suggest that $Z C 3 H 7 B$ $B C O R$ endometrial stromal sarcomas are indeed histologically high-grade.

Although ZC3H7B-BCOR endometrial stromal sarcomas appear histologically high-grade, they are morphologically and immunophenotypically distinct from tumors harboring YWHAE-NUTM2 fusions, the sole type of high-grade endometrial stromal sarcoma currently recognized by the World Health Organization. ${ }^{1}$ The fascicular growth, spindle cells and prominent myxoid matrix of these tumors are not seen in YWHAE-NUTM2 high-grade endometrial stromal sarcomas that are typically composed of sheets, nested, and pseudo-papillary or pseudo-glandular patterns of round cells. Although a low-grade fibrous or fibromyxoid component may be seen in YWHAE-NUTM2 endometrial stromal sarcomas, this phenomenon has not been observed in tumors harboring BCOR rearrangement. Diffuse cyclin D1 and BCOR expression may be present in both types of high-grade endometrial stromal sarcoma, although half of those with BCOR rearrangement show only limited or absent staining for both markers. CD10, ER and PR expression is typically absent in the high-grade round cell component of YWHAE-NUTM2 endometrial stromal sarcomas, whereas CD10 is positive with variable ER and PR staining in tumors with $Z C 3 H 7 B-B C O R$ fusion.

Tumors harboring $Z C 3 H 7 B-B C O R$ fusion are also morphologically and immunophenotypically different from low-grade endometrial stromal sarcomas, particularly those that have fibroblastic, fibromyxoid or myxoid change. Low-grade endometrial stromal sarcoma with variant histologic features typically exhibit foci of conventional low-grade endometrial stromal neoplasia characterized by bland spindle cells with ovoid to fusiform nuclei associated with delicate arterioles resembling proliferative phase endometrial stroma. Even among tumors with extensive fibroblastic, fibromyxoid or myxoid change, the tumor cells show only mild atypia without prominent nucleoli, and the mitotic index is typically low. Fibroblastic low-grade endometrial stromal sarcomas are frequently hypocellular in contrast to $Z C 3 H 7 B$ $B C O R$ endometrial stromal sarcomas. These features contrast with $Z C 3 H 7 B-B C O R$ endometrial stromal sarcomas that exhibit a greater degree of nuclear atypia associated with brisk mitotic activity. Although CD10 is expressed in both low-grade and $Z C 3 H 7 B-B C O R$ endometrial stromal sarcomas, ER and PR staining is seen in the vast majority of lowgrade tumors, ${ }^{18}$ but less than one-third of high-grade endometrial stromal sarcomas with BCOR rearrangement. Diffuse cyclin D1 and/or BCOR expression and absence of ER and PR expression in endometrial stromal sarcoma with fibroblastic, fibromyxoid or myxoid change should prompt consideration of $Z C 3 H 7 B-B C O R$ endometrial stromal sarcoma and confirmatory molecular studies.

$Z C 3 H 7 B-B C O R$ endometrial stromal sarcoma shares remarkable histologic overlap with myxoid leiomyosarcoma. In fact, four of our tumors were diagnosed previously as myxoid leiomyosarcoma and reclassified as endometrial stromal sarcoma only after the gene fusion status was confirmed retrospectively by FISH or next-generation sequencing. $Z C 3 H 7 B-B C O R$ endometrial stromal sarcomas frequently show at least focal deposition of myxoid matrix and collagen in addition to large thick-walled blood vessels that are commonly seen in smooth muscle neoplasia. ${ }^{19}$ However, ovoid nuclei and tongue-like myometrial infiltration are histologic features in favor of endometrial stromal sarcoma over myxoid leiomyosarcoma, which more frequently shows cigar-shaped nuclei and irregular or destructive myometrial invasion. ${ }^{20,21}$ Gross features may also be helpful in this differential diagnosis as myxoid leiomyosarcomas are often gelatinous or mucoid, ${ }^{20,21}$ whereas none of the $Z C 3 H 7 B-B C O R$ endometrial stromal sarcomas with available gross descriptions in our study showed these features. Although two of our patients had lymph node metastases, a feature more common in endometrial stromal sarcomas than leiomyosarcomas, ${ }^{22}$ the small number of patients hinders our ability to draw conclusions regarding their propensity to involve lymph nodes. All ZC3H7B-BCOR endometrial stromal sarcomas in our current and previous studies express CD10 and show either limited or absent staining with smooth muscle markers. ${ }^{12}$ Less than $50 \%$ of our study cohort showed any myogenic differentiation by immunohistochemistry. Among them, only a single tumor demonstrated focal desmin staining, whereas the remaining tumors showed either SMA expression alone or less frequently, concurrent SMA and h-caldesmon positivity without desmin expression. These findings raise a critical issue in the current evaluation of myxoid uterine mesenchymal tumors in which lack of myogenic differentiation by immunohistochemistry has been acceptable in the diagnosis of myxoid leiomyosarcoma if the morphology is characteristic of the entity. In the largest study of uterine myxoid leiomyosarcomas to date, 3 of 30 tumors reportedly showed CD10 expression alone, CD10 with desmin expression only, or CD10 and concurrent SMA and h-caldesmon 
staining, but without desmin immunoreactivity, suggesting that at least a small subset of their cohort may in fact represent unrecognized $Z C 3 H 7 B-B C O R$ endometrial stromal sarcomas. ${ }^{20}$ Our study suggests that absence of desmin expression in any uterine myxoid sarcoma should raise the possibility of $Z C 3 H 7 B-B C O R$ endometrial stromal sarcoma in the differential diagnosis.

Two tumors in our cohort were previously diagnosed as undifferentiated endometrial or uterine sarcoma. Both were additionally noted to have either having uniform cytology or features that may represent a novel variant of high-grade endometrial stromal sarcoma. The morphology of these tumors were no different from the others, and the diagnoses rendered likely reflect the standard terminology used to classify such neoplasms by the World Health Organization $^{1}$ and limited available histologic and genetic data regarding high-grade endometrial stromal sarcomas at that time. Although undifferentiated endometrial sarcoma was previously used to classify highly aggressive tumors that exhibit marked nuclear pleomorphism and destructive myometrial invasion, undifferentiated uterine sarcoma is now the preferred term advocated by the World Health Organization to highlight the lack of histologic resemblance to proliferative phase endometrial stroma. ${ }^{1}$ Growing evidence suggests that undifferentiated uterine sarcomas consist of a morphologically, immunohistochemically and genetically heterogeneous group of tumors, which may be subclassified into pleomorphic and uniform types, ${ }^{23}$ the latter likely including underrecognized high-grade endometrial stromal sarcomas such as those harboring $Y W H A E-$ NUTM2 $2^{5,7}$ and ZC3H7B-BCOR 12 fusions. Rare examples of pleomorphic undifferentiated uterine sarcomas harboring known endometrial stromal sarcoma-related gene fusions have also been reported and likely represent de-differentiation of otherwise low- or high-grade endometrial stromal sarcomas. ${ }^{23,24} \mathrm{FISH}$ and/or next-generation sequencing may also be necessary in the proper subclassification of undifferentiated uterine sarcoma which remains a diagnosis of exclusion.

Finally, adenosarcoma with sarcomatous overgrowth and myxoid inflammatory myofibroblastic tumor may also mimic $Z C 3 H 7 B-B C O R$ endometrial stromal sarcoma. Two of our tumors showed rare benign endometrioid glands; however, none showed periglandular stromal condensation or phyllodes-like architecture that is diagnostic of adenosarcoma. ${ }^{25}$ BCOR genetic abnormalities have also not been detected in adenosarcomas. ${ }^{26}$ Inflammatory myofibroblastic tumors may exhibit myxoid stroma, but these rare neoplasms typically have an inflammatory infiltrate, exhibit ALK expression by immunohistochemistry, and harbor $A L K$ rearrangements. ${ }^{27-29}$ ALK immunohistochemistry was performed on only three of our tumors as part of the diagnostic work-up and was negative in all of them.
Although we did not formally investigate the concordance between cyclin D1 and BCOR expression in all of our tumors, it is of interest that diffuse cyclin D1 is present in $86 \%$ of tumors compared with diffuse BCOR expression in only 50\%, similar to that reported in our recent study evaluating the utility of BCOR expression in high-grade endometrial stromal sarcomas harboring YWHAE rearrangement and BCOR genetic abnormalities. ${ }^{9}$ The mechanism by which BCOR expression is upregulated among tumors with YWHAE and BCOR genetic aberrations is unclear. It is also uncertain why only half of $Z C 3 H 7 B-B C O R$ endometrial stromal sarcomas demonstrate diffuse BCOR staining when there appears to be no correlation between BCOR expression and BCOR breakpoints in the fusion transcripts. ${ }^{9}$ Based on our findings in the current study, we suggest using both cyclin D1 and BCOR along with CD10, desmin, and SMA, when a diagnosis of $Z C 3 H 7 B-B C O R$ endometrial stromal sarcoma is suspected. It should be noted, however, that cyclin D1 and BCOR expression may also be seen in the round cell component of YWHAENUTM2 high-grade endometrial stromal sarcomas, rare low-grade endometrial stromal sarcomas, as well as a small subset of spindle cell leiomyosarcomas, ${ }^{9}$ emphasizing the importance of correlating immunophenotype with morphology when evaluating uterine sarcomas.

The presence of $B C O R$ rearrangements among various tumor types suggests that $B C O R$ has an important role in driving tumorigenesis. The BCL-6 corepressor (BCOR) gene, located on chromosome Xp11.4, encodes BCOR, which interacts with PCGF1 in a variant polycomb repressive complex (PRC1) that enhances transcriptional repression. ${ }^{30,31}$ Germline $B C O R$ mutations have been found in syndromic diseases such as oculo-facio-cardiodental syndrome and Lenz micropthalmia. ${ }^{32}$ Somatic $B C O R$ mutations have been reported in hematologic malignancies, ${ }^{33-36}$ retinoblastoma, ${ }^{37}$ medulloblastoma, ${ }^{38}$ central nervous system primitive neuroectodermal tumors, ${ }^{39}$ rhabdomyosarcoma ${ }^{40}$ and clear cell sarcoma of the kidney. ${ }^{41-43} Z C 3 H 7 B-B C O R$ fusions are not limited to endometrial stromal sarcomas and have been found in a subset of ossifying fibromyxoid tumors, which lack S100 expression and show malignant features. ${ }^{13}$ BCOR rearrangements have also been identified in soft tissue small blue round cell sarcomas and clear cell sarcoma of the kidney, albeit involving the 5' end in contrast to the 3' end that is seen in tumors harboring $Z C 3 H 7 B-B C O R$ fusion. ${ }^{44-47}$ We also recently identified $B C O R$ internal tandem duplications in highgrade endometrial stromal sarcoma, the same genetic aberration detected in soft tissue small blue round cell sarcomas and clear cell sarcoma of the kidney. ${ }^{9}$

Our clinical data are limited because of the rarity of $Z C 3 H 7 B-B C O R$ endometrial stromal sarcomas. Based on the five patients with clinical follow-up at Memorial Sloan Kettering Cancer Center, there is a trend toward a worse prognosis compared with published outcomes of patients with low-grade 
endometrial stromal sarcoma. Prior studies indicating aggressive clinical behavior among patients with YWHAE-NUTM2 high-grade endometrial stromal sarcoma also suffer from similar limitations given small sample size and referral bias. ${ }^{2,4}$ Multiinstitutional studies are required for comparative analysis of clinical behavior among patients with ZC3H7B-BCOR, YWHAE-NUTM2 high-grade and low-grade endometrial stromal sarcomas.

We propose that $Z C 3 H 7 B-B C O R$ endometrial stromal sarcomas be included among the highgrade category of endometrial stromal sarcomas given the available morphologic evidence and limited clinical data. These tumors display highgrade spindle cell histology frequently associated with myxoid matrix and are positive for CD10 with either limited or absent evidence of smooth muscle differentiation by immunohistochemistry. $Z C 3 H 7 B$ $B C O R$ endometrial stromal sarcomas should be distinguished from other myxoid uterine mesenchymal tumors given the implications for patient management.

\section{Disclosure/conflict of interest}

The authors declare no conflict of interest.

\section{References}

1 Oliva E, Carcangiu ML, Carinelli SG, et al. Mesenchymal tumors. In: Kurman RJ, Carcangiu ML, Herrington CS, Young RH eds. WHO Classification of Tumours of Female Reproductive Organs 4th edn. International Agency for Research on Cancer: Lyon, France, 2014, pp 135-147.

2 Lee $\mathrm{CH}, \mathrm{Ou}$ WB, Marino-Enriquez A, et al. 14-3-3 Fusion oncogenes in high-grade endometrial stromal sarcoma. Proc Natl Acad Sci USA 2012;109:929-934.

3 Conklin CM, Longacre TA. Endometrial stromal tumors: the new WHO classification. Adv Anat Pathol 2014;21:383-393.

4 Lee CH, Marino-Enriquez A, Ou W, et al. The clinicopathologic features of YWHAE-FAM22 endometrial stromal sarcomas: a histologically high-grade and clinically aggressive tumor. Am J Surg Pathol 2012;36:641-653.

5 Croce S, Hostein I, Ribeiro A, et al. YWHAE rearrangement identified by FISH and RT-PCR in endometrial stromal sarcomas: genetic and pathological correlations. Mod Pathol 2013;26:1390-1400.

6 Aisagbonhi O, Harrison B, Zhao L, et al. YWHAE rearrangement in a purely conventional low-grade endometrial stromal sarcoma that transformed over time to high-grade sarcoma: importance of molecular testing. Int J Gynecol Pathol (in press); e-pub ahead of print.

7 Sciallis AP, Bedroske PP, Schoolmeester JK, et al. Highgrade endometrial stromal sarcomas: a clinicopathologic study of a group of tumors with heterogenous morphologic and genetic features. Am J Surg Pathol 2014;38:1161-1172.

8 Lee CH, Ali RH, Rouzbahman M, et al. Cyclin D1 as a diagnostic immunomarker for endometrial stromal sarcoma with YWHAE-FAM22 rearrangement. Am J Surg Pathol 2012;36:1562-1570.

9 Chiang S, Lee CH, Stewart CJR, et al. BCOR is a robust diagnostic immunohistochemical marker of genetically diverse high-grade endometrial stromal sarcoma, including tumors exhibiting variant morphology. Mod Pathol 2017;30:1251-1261.

10 Kruse AJ, Croce S, Kruitwagen RF, et al. Aggressive behavior and poor prognosis of endometrial stromal sarcomas with YWHAE-FAM22 rearrangement indicate the clinical importance to recognize this subset. Int J Gynecol Pathol 2014;24:1616-1622.

11 Gremel G, Liew M, Hamzei F, et al. A prognosis based classification of undifferentiated uterine sarcomas: identification of mitotic index, hormone receptors and YWHAE-FAM22 translocation status as predictors of survival. Int J Cancer 2015;136:1608-1618.

12 Hoang LN, Aneja A, Conlon N, et al. Novel high-grade endometrial stromal sarcoma: a morphologic mimicker of myxoid leiomyosarcoma. Am J Surg Pathol 2017;41: 12-24.

13 Antonescu CR, Sung YS, Chen CL, et al. Novel ZC3H7B-BCOR, MEAF6-PHF1, and EPC1-PHF1 fusions in ossifying fibromyxoid tumors-molecular characterization shows genetic overlap with endometrial stromal sarcoma. Genes Chromosomes Cancer 2014;53: 183-193.

14 Panagopoulos I, Thorsen J, Gorunova L, et al. Fusion of the ZC3H7B and BCOR genes in endometrial stromal sarcomas carrying an X;22-translocation. Genes Chromosomes Cancer 2013;52:610-618.

15 Cheng DT, Mitchell TN, Zehir A, et al. Memorial Sloan Kettering-Integrated Mutation Profiling of Actionable Cancer Targets (MSK-IMPACT): a hybridization capture-based next-generation sequencing clinical assay for solid tumor molecular oncology. J Mol Diagn 2015;17:251-264.

16 Kao YC, Sung YS, Zhang L, et al. Recurrent BCOR internal tandem duplication and YWHAE-NUTM2B fusions in soft tissue undifferentiated round cell sarcoma of infancy: overlapping genetic features with clear cell sarcoma of kidney. Am J Surg Pathol 2016;40: 1009-1020.

17 Zheng Z, Liebers M, Zhelyazkova B, et al. Anchored multiplex PCR for targeted next-generation sequencing. Nat Med 2014;20:1479-1484.

18 Chiang S, Oliva E. Recent developments in uterine mesenchymal neoplasms. Histopathology 2013;62: 124-137.

19 Goh R, Dal Cin P, Chiang S, et al. Myxoid smooth muscle tumors of the uterus: clinicopathologic study of 40 cases. Mod Pathol 2016;29:285A.

20 Parra-Herran C, Schoolmeester JK, Yuan L, et al. Myxoid leiomyosarcoma of the uterus: a clinicopathologic analysis of 30 cases and review of the literature with reappraisal of its distinction from other uterine myxoid mesenchymal neoplasms. Am J Surg Pathol 2016;40:285-301.

21 King ME, Dickersin GR, Scully RE. Myxoid leiomyosarcoma of the uterus. A report of six cases. Am J Surg Pathol 1982;6:589-598.

22 Gadducci A, Cosio S, Romanini A, et al. The management of patients with uterine sarcoma: a debated clinical challenge. Crit Rev Oncol Hematol 2008;65: 129-142.

23 Kurihara S, Oda Y, Ohishi Y, et al. Endometrial stromal sarcomas and related high-grade sarcomas: 
immunohistochemical and molecular genetic study of 31 cases. Am J Surg Pathol 2008;32:1228-1238.

24 Koontz JI, Soreng AL, Nucci M, et al. Frequent fusion of the JAZF1 and JJAZ1 genes in endometrial stromal tumors. Proc Natl Acad Sci USA 2001;98: 6348-6353.

25 Pinto A, Howitt B. Uterine adenosarcoma. Arch Pathol Lab Med 2016;140:286-290.

26 Piscuoglio S, Burke KA, Ng CK, et al. Uterine adenosarcomas are mesenchymal neoplasms. J Pathol 2016;238:381-388.

27 Parra-Herran C, Quick CM, Howitt BE, et al. Inflammatory myofibroblastic tumor of the uterus: clinical and pathologic review of 10 cases including a subset with aggressive clinical course. Am J Surg Pathol 2015;39: 157-168.

28 Fuehrer NE, Keeney GL, Ketterling RP, et al. ALK-1 protein expression and ALK gene rearrangements aid in the diagnosis of inflammatory myofibroblastic tumors of the female genital tract. Arch Pathol Lab Med 2012;136:623-626.

29 Bennett JA, Nardi V, Rouzbahman M, et al. Inflammatory myofibroblastic tumor of the uterus: a clinicopathological, immunohistochemical, and molecular analysis of 13 cases highlighting their broad morphologic spectrum. Mod Pathol 2017;30:1489-1503.

30 Huynh KD, Fischle W, Verdin E, et al. BCoR, a novel corepressor involved in BCL-6 repression. Genes Dev 2000;14:1810-1823.

31 Fan Z, Yamaza T, Lee JS, et al. BCOR regulates mesenchymal stem cell function by epigenetic mechanisms. Nat Cell Biol 2009;11:1002-1009.

32 Ng D, Thakker N, Corcoran CM, et al. Oculofaciocardiodental and Lenz microphthalmia syndromes result from distinct classes of mutations in BCOR. Nat Genet 2004;36:411-416.

33 Grossmann V, Tiacci E, Holmes AB, et al. Wholeexome sequencing identifies somatic mutations of BCOR in acute myeloid leukemia with normal karyotype. Blood 2011;118:6153-6163.

34 Lindsley RC, Mar BG, Mazzola E, et al. Acute myeloid leukemia ontogeny is defined by distinct somatic mutations. Blood 2015;125:1367-1376.

35 Damm F, Chesnais V, Nagata Y, et al. BCOR and BCORL1 mutations in myelodysplastic syndromes and related disorders. Blood 2013;122:3169-3177.

36 Dobashi A, Tsuyama N, Asaka R, et al. Frequent BCOR aberrations in extranodal NK/T-Cell lymphoma, nasal type. Genes Chromosomes Cancer 2016;55: 460-471.

37 Zhang J, Benavente CA, McEvoy J, et al. A novel retinoblastoma therapy from genomic and epigenetic analyses. Nature 2012;481:329-334.

38 Pugh TJ, Weeraratne SD, Archer TC, et al. Medulloblastoma exome sequencing uncovers subtype-specific somatic mutations. Nature 2012;488:106-110.

39 Sturm D, Orr BA, Toprak UH, et al. New brain tumor entities emerge from molecular classification of CNSPNETs. Cell 2016;164:1060-1072.

40 Shern JF, Chen L, Chmielecki J, et al. Comprehensive genomic analysis of rhabdomyosarcoma reveals a landscape of alterations affecting a common genetic axis in fusion-positive and fusion-negative tumors. Cancer Discov 2014;4:216-231.

41 Astolfi A, Melchionda F, Perotti D, et al. Whole transcriptome sequencing identifies BCOR internal tandem duplication as a common feature of clear cell sarcoma of the kidney. Oncotarget 2015;6: 40934-40939.

42 Karlsson J, Valind A, Gisselsson D. BCOR internal tandem duplication and YWHAE-NUTM2B/E fusion are mutually exclusive events in clear cell sarcoma of the kidney. Genes Chromosomes Cancer 2016;55: 120-123.

43 Ueno-Yokohata H, Okita H, Nakasato K, et al. Consistent in-frame internal tandem duplications of BCOR characterize clear cell sarcoma of the kidney. Nat Genet 2015;47:861-863.

44 Argani P, Kao YC, Zhang L, et al. Primary renal sarcomas with BCOR-CCNB3 gene fusion: a report of 2 cases showing histologic overlap with clear cell sarcoma of kidney, suggesting further link between BCOR-related sarcomas of the kidney and soft tissues. Am J Surg Pathol 2017; 41: 1702-1712.

45 Specht K, Zhang L, Sung YS, et al. Novel BCORMAML3 and ZC3H7B-BCOR gene fusions in undifferentiated small blue round cell sarcomas. Am J Surg Pathol 2016;40:433-442.

46 Peters TL, Kumar V, Polikepahad S, et al. BCORCCNB3 fusions are frequent in undifferentiated sarcomas of male children. Mod Pathol 2015;28:575-586.

47 Li WS, Liao IC, Wen MC, et al. BCOR-CCNB3-positive soft tissue sarcoma with round-cell and spindle-cell histology: a series of four cases highlighting the pitfall of mimicking poorly differentiated synovial sarcoma. Histopathol 2016;69:792-801. 